\title{
Comparison of Methods for Fullerene Detection and Measurements of Reactive Oxygen Production in Cosmetic Products
}

\author{
So-Ryong Chae, ${ }^{1,2, \dagger}$ Ernest M. Hotze, ${ }^{2,3, \dagger}$ Yao Xiao,, Jerome Rose, ${ }^{1,3}$ and Mark R. Wiesner ${ }^{1,2, \star}$ \\ ${ }^{1}$ Department of Civil and Environmental Engineering, Pratt School of Engineering, Duke University, Durham, North Carolina. \\ ${ }^{2}$ Center for the Environmental Implications of Nanotechnology (CEINT) and the International Consortium \\ for the Environmental Implications of NanoTechnology (iCEINT), Durham, NC and Aix en Provence, France. \\ ${ }^{3}$ CEREGE, UMR 6635 CNRS/UPC, Aix en Provence, France.
}

Received: April 2, $2010 \quad$ Accepted in revised form: June 7, 2010

\begin{abstract}
Numerous commercial products incorporate novel engineered nanomaterials such as gold, silica, zinc oxide, and fullerenes in complex matrices such as polymer composites, creams, and textiles. Analytical methods for detecting nanomaterials in complex matrices are not well developed. Moreover, nanomaterial content and properties of these commercial products are typically unknown and protected for proprietary reasons. This study had two primary aims: detection of $\mathrm{C}_{60}$ within commercial face creams to establish a baseline concentration in these products (the first time this has been performed) and detection of residual $\mathrm{C}_{60}$ reactivity remaining in the products aged in water under various light conditions with a view toward environmental exposure assessment. To achieve these aims, three commercial creams advertised as containing the fullerene nanomaterials were investigated using a range of analytical techniques. Among the detection methods tested, only extraction followed by high-performance liquid chromatography was able to detect fullerenes in these products. The measured quantities of $\mathrm{C}_{60}$ in these creams represented $<0.005 \%(\mathrm{w} / \mathrm{w})$ with an unknown yield because total amounts added to the creams were unknown. Production of reactive oxygen species from these face creams was measured after aging them in water as well as exposing them to solar spectrum illumination or ultraviolet light, or storage in the dark. Singlet oxygen generated in the products after $48 \mathrm{~h}$ of aging was correlated with the amounts of $\mathrm{C}_{60}$ extracted from preaged samples, indicating residual photochemical reactivity and pointing toward the long-term impacts of utilizing these materials in commercial products.
\end{abstract}

Key words: nanomaterials; commercial creams; fullerene; production of reactive oxygen species; aging

\section{Introduction}

A VARIETY OF commercial products containing novel engineered nanomaterials are being marketed and include products such as silver-coated fabrics designed to resist microbes (Tweden et al., 1997; Sachinvala et al., 2007) and "antiaging" face creams with fullerene as an active ingredient to destroy free radicals (Cosmetic Dermatology, 2009; Naturelle, 2009). Although the environmental impacts of engineered nanomaterials remain largely unknown (Wiesner et al., 2006), the introduction of these types of nanomaterial-enabled products is evolving at a pace that will likely exceed the ability to assess and regulate these materials. From a life-cycle perspective it is essential that data on nanomaterial content of products be disseminated to the risk assessment community

\footnotetext{
†These two authors contributed equally to this work.

${ }^{*}$ Corresponding author: Department of Civil and Environmental Engineering, Pratt School of Engineering, Duke University, Durham, NC 27708. Phone: 919-660-5292; Fax: 919-660-5219; E-mail: wiesner@ duke.edu
}

to estimate exposure to nanomaterials from various sources. As carbon-based nanomaterials become more prevalent in commercial products, it is particularly important to understand how to detect the degradation products of these materials as well as to determine if these products remain as reactive as the original nanomaterials (Isaacson et al., 2009). Therefore, the current work considers the use of fullerenes in cosmetics and the potential toxic effect of reactive oxygen species (ROS) produced by the cosmetic products after use.

Early reports of fullerene toxicity to both bacterial (Fortner et al., 2005; Lyon et al., 2006, 2008; Fang et al., 2007; Chae et al., $2009 \mathrm{~b}$ ) and human cell cultures (Xia et al., 2006; Roberts et al., 2008) use methodologies and organism specificities that as of now do not produce a universal indication of what may be the wide-ranging impacts of these materials. However, Lyon et al. (2008) have recently put forth a more general toxicity hypothesis pointing to the electron affinity of $\mathrm{C}_{60}$ altering the electron transport chain at the surface of the cell wall in bacteria and therefore disrupting normal cell respiration and eventually leading to death. Interestingly, this electron affinity of $\mathrm{C}_{60}$ or "radical sponge fullerene" is the same property 
that led to incorporating it into antiaging commercial cream products.

In addition to toxicity effects in the absence of light, fullerene derivatives and fullerenes can be photoactivated in aqueous environments to produce ROS such as superoxide $\left(\mathrm{O}_{2}{ }^{--}\right)$and singlet oxygen $\left({ }^{1} \mathrm{O}_{2}\right)$ via type I and type II photosensitization pathways given appropriate suspension conditions (Pickering and Wiesner, 2005; Hotze et al., 2008). Photosensitized production of ROS does not appear to be associated with bacterial inactivation (Lyon et al., 2008). ROS generation by fullerene derivatives, however, has been shown to impact bacteriophages by inactivating them at much higher rates than those found for irradiation alone (Badireddy et al., 2007). As these creams are of proprietary composition, information on the $\mathrm{C}_{60}$ content is largely lacking and must be surmised from measurements of the total amount of detectable fullerene present in the products. Measurements of ROS generation by dispersed creams might also provide information on $\mathrm{C}_{60}$ content, although interferences with other materials present in the creams (e.g., antioxidants or surfactants) and the aggregation state of $\mathrm{C}_{60}$ may reduce ROS generation and therefore conceal or obscure the presence of $C_{60}$. Finally, changes in $\mathrm{C}_{60}$ properties over time due to aging of the material might also affect ROS generation, transport properties, and impacts on organisms.

This study has two primary aims: detection of fullerene within commercial face creams to establish for the first time a baseline concentration in these products and to quantify residual fullerene reactivity remaining in the products aged in water under various conditions of illumination. Both of these aims were performed with a broader view toward the environmental exposure assessment of the use of nanomaterials in commercial products. Quantitative measurements of fullerene in commercial products and various environmental samples are not fully developed even though some previous studies have reported measurement of $\mathrm{C}_{60}$ in biological and geological samples (Moussa et al., 1997; Jehlicka et al., 2005; Isaacson et al., 2009), as well as aqueous matrices (Bouchard and Ma, 2008; Chen et al., 2008). Extraction of $\mathrm{C}_{60}$ essentially involves organic solvent (e.g., toluene) followed by chromatographic separation and appropriate detection method. Of the detection methods attempted, mass spectrometry achieves the lowest detection limit, but with the highest costs and lowest operating efficiency. Alternatively, ultraviolet (UV) spectroscopy offers a cheaper and more efficient alternative with an acceptable detection limit (Bouchard and Ma, 2008; Chen et al., 2008). In this study, four methods were evaluated for detecting $\mathrm{C}_{60}$ in these products: Fourier transform infrared spectroscopy (FTIR), scanning electron microscopy with energy-dispersive $X$-ray spectroscopy (SEMEDX), carbon-13 NMR ( ${ }^{13} \mathrm{C}$ NMR), and high-performance liquid chromatography (HPLC). We observed that only the method of extraction into toluene followed by HPLC was able to detect what appear to be extremely small amounts of $C_{60}$ present in the creams. Creams were then aged in water under solar spectrum irradiation, UV-A irradiation, and dark conditions, each resulting in three separate layers of aged product. This was followed by ROS detection of superoxide by XTT [2,3-bis(2-methoxy-4-nitro-5-sulfophenyl)-2H-tetrazolium5-carboxanilide; reduction followed by spectrophotometric detection] or detection of singlet oxygen by Singlet Oxygen Sensor Green (SOSG; reaction followed by fluores- cence detection) in UV-A irradiated samples of the aged products.

\section{Experimental Protocols}

\section{Chemicals and products}

Three commercial face creams were purchased and labeled as products $\mathrm{A}, \mathrm{B}$, and $\mathrm{C}$ (see Table 1 for detailed information). $\mathrm{C}_{60}\left(99.9 \%\right.$ pure) and hydroxylated $\mathrm{C}_{60}$ [fullerol, $\mathrm{C}_{60}(\mathrm{OH})_{24}$ ] were purchased from MER (Tucson, AZ). Superoxide dismutase (SOD) (bovine ethrocytes), $\beta$-carotene, and XTT were obtained from Sigma-Aldrich (St. Louis, MO). SOSG was purchased from Molecular Probes-Invitrogen (Carlsbad, CA). Double deionized (DI) water had resistivity of $>18.2 \mathrm{M} \Omega \mathrm{cm}$ and dissolved organic carbon concentration was $<10 \mu \mathrm{g} / \mathrm{L}$.

\section{Characterization of $C_{60}$ in face creams}

Samples were analyzed directly by FTIR, SEM-EDX, and ${ }^{13} \mathrm{C}$ NMR without any aging processes being applied. FTIR was performed with an Equinox 55 Bruker spectrometer. Emitted light spectra were recorded in the midinfrared spectra in the range $2,100-400 \mathrm{~cm}^{-1}$ at a resolution of $4 \mathrm{~cm}^{-1}$ with 32 scans per spectrum. All scans were made by dehydrating $\sim 1 \mathrm{mg}$ of each cream at $60^{\circ} \mathrm{C}$ overnight and homogenizing the resulting oil with $30 \mathrm{mg}$ crushed $\mathrm{KBr}$, which was pelleted before analysis. SEM imagery coupled with X-ray analysis provided information on the elemental composition of the three products. ${ }^{13} \mathrm{C}$ NMR was performed with an Avance 400 WB Bruker spectrometer. Spectra were obtained with highresolution magic angle spinning $(1 \mathrm{kHz})$ and ${ }^{1} \mathrm{H}$ decoupling. A pulse at $100.7 \mathrm{MHz}\left({ }^{13} \mathrm{C}\right)$ was applied at $30^{\circ}$. The acquisition time was $270 \mathrm{~ms}$, with a $2 \mathrm{~s}$ recycling time between acquisitions and a total of 80,000 scans.

The ability to extract fullerene aggregates from an aqueous matrix to toluene was then evaluated. About $3 \mathrm{~g}$ of each product was placed in a glass vial containing $10 \mathrm{~mL}$ DI water with $20 \mathrm{mM} \mathrm{KCl}$ before the addition of $20 \mathrm{~mL}$ of toluene. Preliminary experiments optimized the concentration of $\mathrm{KCl}$ and volume ratio between water and toluene (data not shown). Then, the vial was vertically mixed overnight and the supernatant containing fullerene was further analyzed using an HPLC. For quantitative analysis of fullerene, a standard curve was developed using known concentrations of $\mathrm{C}_{60}$ in toluene. From the HPLC analysis, it was found that fullerene in toluene has two distinguishing peaks at 285 and $334 \mathrm{~nm}$, with a retention time of $7.7 \mathrm{~min}$. A linear relationship between concentrations of fullerene in toluene and absorbance at $285 \mathrm{~nm}$ (peak area) was observed over the concentration range from $15 \mathrm{ppb}$ to $15 \mathrm{ppm}$ (data not shown). Lastly, quantitative analysis of fullerene was performed using an HPLC (ProStar, Varian, Palo Alto, CA) equipped with a Cosmosil Buckyprep column (Nacalai USA, San Diego, CA). The chromatographic separation was performed in isocratic mode at a constant flow rate of $1 \mathrm{~mL} / \mathrm{min}$ with a mobile phase of $100 \%$ toluene at $285 \mathrm{~nm}$ UV wavelength. Comparison of resulting spectra to the calibration curve allowed for the fullerene concentration to be determined by peak area. To evaluate recovery rate, different amounts of fullerene were spiked into product B and tested by the protocol described. 
Table 1. Characteristics of Commercial Face Creams Tested

\begin{tabular}{|c|c|c|}
\hline Product (Origin) & Function & Ingredient \\
\hline A (United States) & $\begin{array}{l}\text { Reduce the } \\
\text { formation } \\
\text { of lines on } \\
\text { the face }\end{array}$ & $\begin{array}{l}\text { Water (AQUA), methylsilanol hydroxyprline aspartate, cyclomethicone, } \\
\text { C12-15 alkyl lactate, acrylamide/ammonium acrylate copolymer, caprylyl } \\
\text { trimethicone, octyldodecyl stearate, urea, soybean germ extract, myristyl } \\
\text { myristate, diisopropyl adipate, dicaprylyl carbonate, sodium hyaluronate, } \\
\text { methyl methacrylate crosspolymer, cetyl palmitate, phenoxyethanol, } \\
\text { butylene glycol, PEG-100 stearate, glyceryl stearate sorbitan stearate } \\
\text { ammonium, acryloyldimethyltaurate/VP copolymer, TIMP-2, sodium } \\
\text { stearyl fumarate, magnesium silicate, PEG-40 stearate, panthenol, } \\
\text { fullerenes, glycerin, lecithin, allantoin, lavender oil, himanthalia elongata } \\
\text { extract, methylparaben, camellia sinensis leaf extract, disodium EDTA, } \\
\text { castoryl maleate, BHT, grape seed extract, hydrolyzed collagen, silanediol } \\
\text { salicylate, sodium styrene/acrylates copolymer, triethanolamine, } \\
\text { hydrogenated lecithin, ethylparaben, butylparaben, alcohol, } \\
\text { propylparaben, isobutylparaben, magnesium ascorbyl phosphate, } \\
\text { silanetriol }\end{array}$ \\
\hline B (Japan) & $\begin{array}{l}\text { Avoid black } \\
\text { spots and } \\
\text { wrinkles }\end{array}$ & $\begin{array}{l}\text { Water, triethylhexanoin, glycerin, squalane, stearic acid, pentylene glycol, } \\
\text { baty alcohol, glyceryl stearate, jojoba oil, behenyl alcohol, bamboo } \\
\text { essence, fullerene, hyaluronan, cherry blossoms extract, hypericum } \\
\text { flowers extract, mulberry extract, light galangal leaf extract, green tea } \\
\text { extract, panax ginseng root extract, paeonia albiflora extract, saxifraga } \\
\text { stolonifera extract, SOD, cyanocobalamin, tocopherol, carbomer, sodium } \\
\text { hydroxide, lecithin, glycerine acid ester, htdroxyethyl cellulose, lavender } \\
\text { extract, grapefruit seed extract }\end{array}$ \\
\hline $\begin{array}{l}\text { C (United } \\
\text { Kingdom) }\end{array}$ & $\begin{array}{l}\text { Eliminate } \\
\text { damaging } \\
\text { free radicals }\end{array}$ & $\begin{array}{l}\text { Vitamin A, vitamin C, vitamin E, coenzyme Q10, alpha-lipoic acid, } \\
\text { fullerene, antioxidant with anti-inflammatory properties green tea, D- } \\
\text { pathenol, SOD, bisabolol (camomile derivative), lactoperoxidase, } \\
\text { carnosine, pycnogenol (extract from pine bark), gingko biloba }\end{array}$ \\
\hline
\end{tabular}

EDTA, ethylenediaminetetraacetic acid; SOD, superoxide dismutase; PEG, polyethylene glycol; TIMP, tissue inhibitors of metalloproteinases; $\mathrm{BH}$, butylated hydroxytoluene.

\section{Aging process}

Cream samples stored in their original containers are referred to in this study as "unaged" creams. Aging of the three face creams was performed by addition of $3 \mathrm{mg}$ of unaged cream into $50 \mathrm{~mL}$ of ultrapure water and stirring for $48 \mathrm{~h}$. Three illumination conditions were tested for each of the three creams: covered (dark); UV-A lamps (365 nm); and a lamp meant to mimic the solar spectrum. Low-pressure UV-A irradiation was done in the presence of two $15-\mathrm{W}$ fluorescent UV bulbs (Philips TLD $15 \mathrm{~W} / 08$ ). These bulbs have an output spectrum peak at $365 \mathrm{~nm}$ (ranging from 354 to $396 \mathrm{~nm}$ ) and a total irradiance of $24.1 \mathrm{~W} / \mathrm{m}^{2}$ (Pickering and Wiesner, 2005). The solar spectrum irradiation was done in the presence of one 400-W lamp (Philips Mater HPI-T Plus 400 W/645 E40 SLV). This bulb had an output spectrum ranging from 300 to $900 \mathrm{~nm}$, with a total irradiance of $72.19 \mathrm{~W} / \mathrm{m}^{2}$. Aged cream was separated into three distinct layers. The top layer of the aged sample was nonaqueous, could be separated easily, and is referred to as the surface layer. Aqueous aged material remaining below the top layer was further separated by centrifugation at $10,000 \mathrm{rpm}$ for $1 \mathrm{~h}$. The resultant supernatant (stable layer) remaining in suspension was analyzed separately from the resuspended, centrifuged pellet.

\section{Detection of ROS in face creams}

SOSG was used to measure ${ }^{1} \mathrm{O}_{2}$ concentrations (Flors et al., 2006) in irradiated aged samples of three fullerene cosmetic products. SOSG was first prediluted in $33 \mu \mathrm{L}$ methanol and ultrapure water to $165 \mu \mathrm{M}$ as recommended by the manufacturer and then diluted 10 -fold to a final concentration of $16.5 \mu \mathrm{M}$ before measurement. Suspensions were placed in a 25 -mL Petri dish with $\sim 20 \mathrm{~cm}^{2}$ surface area. Samples of $1 \mathrm{~mL}$ were taken at every $5 \mathrm{~min}$ for $30 \mathrm{~min}$ in the dark. The fluorescence units (a.u.) from this dark measurement were read as a background (Modulus Single Tube 9200; Turner Biosystems, Sunnyvale, CA) and subtracted from readings taken at every $5 \mathrm{~min}$ for $30 \mathrm{~min}$ once UV-A lamps were switched on (Philips TLD 15 W/08). Fluorescence intensity could then be correlated with relative production of singlet oxygen by unaged and aged cream products.

XTT reduction was employed to measure the production of superoxide. The reduction of XTT results in an increase in optical density at $470 \mathrm{~nm}$, which can be used to quantify the relative amount of superoxide present (Ukeda et al., 1997; Bartosz, 2006). The concentration of superoxide was determined by comparing XTT reduction with and without a quencher for superoxide, SOD, which allowed nonsuperoxide-related XTT reactions to be accounted for. SOD-containing samples served to eliminate the influence of background absorbance of suspensions at $470 \mathrm{~nm}$. Optical density could then be correlated with relative production of superoxide by unaged and aged cream products. Here, we compare photochemical reactivity of the cosmetic products with those of fullerol, which is a wellknown ROS generator (Pickering and Wiesner, 2005). Fullerol suspension (a positive control) was prepared by adding the 
powdered form of these materials to the DI water (Chae et al., 2009a). The ideal negative control for ROS (identical composition minus the $\mathrm{C}_{60}$ ) was not available because of the proprietary nature of the creams. All experiments were performed in triplicate and standard deviations are included. Student's $t$-test was used to assess the significance of the results with a $95 \%$ confidence interval.

\section{Results and Discussion}

\section{SEM-EDX, FTIR, and ${ }^{13} \mathrm{C}$ NMR measurements}

SEM technique coupled with element analysis (EDX) provided general information about carbon and particle content for the unmodified products. From this analysis, it was found that carbon compounds were indeed present with other elemental spectra (data not shown). But, this information (along with SEM images) is limited because it is difficult to distinguish nano-based carbon material from other forms found in cream ingredients. We subsequently explored the use FTIR and ${ }^{13} \mathrm{C}$ NMR techniques as a basis for detecting the presence of $\mathrm{C}_{60}$ in these cosmetics.

All three products were analyzed by FTIR following partial dehydration. However, even after the dehydration step, samples contained at least $50 \%$ water by volume. FTIR spectra for all three products did not include the major bands $(1,426$ 1,429 and $1,179-1,181 \mathrm{~cm}^{-1}$ ) attributed to the icosahedral symmetry of the $\mathrm{C}_{60}$ molecule (Treubig and Brown, 2002) (data not shown). However, these measurements do not rule out the possibility that $\mathrm{C}_{60}$ molecules were present at lower concentrations as the $C_{60}$ may have been obscured by the presence of other molecules (e.g., molecules containing $\mathrm{C}=\mathrm{C}$ bonds absorbing near $1,460 \mathrm{~cm}^{-1}$ ) in the cream matrix. ${ }^{13} \mathrm{C}$ NMR with ${ }^{1} \mathrm{H}$ decoupling was also performed with the goal of obtaining a qualitative picture of fullerenes present relative to other carbon molecules in the cream matrix of product A. Significant amounts of carbon from fullerene present in the sample would be indicated by the appearance of a distinct single peak around 143 ppm (Yannoni et al., 1991; Fortner et al., 2005). But, in the case of product A, no such peak could be differentiated from the background of the spectrum (data not shown). Because of the inability to distinguish $\mathrm{C}_{60}$ from other carbon-based molecules by ${ }^{13} \mathrm{C}$ NMR, SEM-EDX, or FTIR in the cream matrix of product $A$, subsequent characterization of products $\mathrm{A}, \mathrm{B}$, and $\mathrm{C}$ was limited to extraction and HPLC analysis of $\mathrm{C}_{60}$ in these creams.

\section{Quantitative analysis of $C_{60}$ in face creams by HPLC}

For the quantitative analysis of $\mathrm{C}_{60}$ by HPLC, different amounts of pure fullerene were spiked into product $B$ by the protocol described and recovery rate was evaluated. As shown in Table 2, when $20 \mathrm{mg}$ of $\mathrm{C}_{60}$ was spiked into $2 \mathrm{~g}$ of the product $(1 \%, \mathrm{w} / \mathrm{w}), 86 \%$ of recovery rate was achieved (run 1). In run 2 , the recovery rate slightly decreased to $82 \%$ when $2 \mathrm{mg}$ of $\mathrm{C}_{60}$ was spiked into $20 \mathrm{~g}$ of the product $(0.01 \%$, $\mathrm{w} / \mathrm{w})$ and the detected $\mathrm{C}_{60}$ concentration $(8.2 \mathrm{mg} / \mathrm{L})$ of the spiked sample by HPLC was close to detected $\mathrm{C}_{60}$ concentrations $(2.6-6.8 \mathrm{mg} / \mathrm{L})$ in the products (Table 3). However, these recovery rates were slightly lower than that from the DI water without product B (in this case, about $90 \%$ of the spiked fullerene was recovered by the first extraction). This $8 \%$ loss in recovery rates was probably due to amphiphilic organic macromolecules such as stearic acid in the face cream acting as a de facto surfactant and stabilizing fullerenes in water, thereby decreasing the extraction efficiency from water to toluene. However, the recovery rate increased to above $95 \%$ with a second toluene extraction with fresh solvent. Finally, small quantities of fullerene were detected at levels of $<0.005 \%(w / w)$ in all products by HPLC with the described protocol.

\section{ROS production of unaged and aged cream products}

XTT formazan adsorption was monitored under UV light to study superoxide production from the three unaged parts (i.e., surface layer, stable, and centrifuged pellet) of each face cream (three samples in triplicate). Superoxide production from unaged samples is summarized in Fig. 1A. The XTT formazan production rate, indicating superoxide production, was slightly higher in samples from surface and stable layers compared with that observed for samples from centrifuged pellets. When $5 \mathrm{mg} / \mathrm{L}$ of fullerol was added to the unaged samples (preseparation), no additional superoxide generation was observed (Fig. 1B).

Aged creams were tested after three different aging processes (i.e., in the dark, or with solar spectrum or UV-A) followed by separation (nine samples in triplicate). As shown in Fig. 2, the superoxide generation of all aged samples is negligible, indicating there was no measurable generation of superoxide. In the presence of oxygen and light, fullerene produces singlet oxygen via a type II pathway and may also produce the superoxide radical via a type I pathway if an appropriate electron donor is present (Hotze et al., 2008). Therefore, we conclude that solution conditions in the cream samples are not appropriate for the generation of superoxide (i.e., no reducing agent is present to enable the transfer of an electron to oxygen).

On the other hand, Fig. 3A shows that SOSG detects significant production of singlet oxygen from unaged face creams. Adding $5 \mathrm{mg} / \mathrm{L}$ of fullerol, the singlet oxygen pro-

Table 2. Quantification of Fullerene Spiked into the Cosmetic Product

\begin{tabular}{|c|c|c|c|c|c|c|}
\hline \multirow[b]{2}{*}{ Sample } & \multirow[b]{2}{*}{$\begin{array}{c}\text { Amount of } C_{60} \text { spiked into } \\
\text { product } B(\%, w / w)\end{array}$} & \multirow{2}{*}{$\begin{array}{c}C_{60} \text { concentration } \\
\text { in water suspension } \\
(m g / L)\end{array}$} & \multicolumn{2}{|c|}{ Detected $C_{60}$ by HPLC $(m g / L)$} & \multicolumn{2}{|c|}{ Recovery rate $(\%)^{\mathrm{a}}$} \\
\hline & & & $\begin{array}{l}\text { After 1st } \\
\text { extraction }\end{array}$ & $\begin{array}{l}\text { After } 2 \text { nd } \\
\text { extraction }\end{array}$ & $\begin{array}{l}\text { After 1st } \\
\text { extraction }\end{array}$ & $\begin{array}{l}\text { After } 2 \text { nd } \\
\text { extraction }\end{array}$ \\
\hline Run 1 & 1 & 200 & 160.3 & 174.6 & 78 & 86 \\
\hline Run 2 & 0.01 & 10 & 10.1 & 10.8 & 75 & 82 \\
\hline
\end{tabular}

${ }^{a}$ Originally, product B contains fullerene $(2.6 \mathrm{mg} / \mathrm{L}$; see Table 3$)$. When recovery rate was calculated, the background fullerene concentration in product B was subtracted from the detected amount in the fourth column in this table. 
Table 3. Quantification of Fullerene in Three Cosmetic Products

\begin{tabular}{|c|c|c|c|c|}
\hline Sample & Amount (g) & $\begin{array}{c}\text { Detected } C_{60} \\
\text { by } H P L C \\
(m g / L)\end{array}$ & $\begin{array}{l}\text { Amount of } C_{60} \\
\text { in sample }(m g)\end{array}$ & $\begin{array}{l}\mathrm{C}_{60} \text { in } \\
\text { product } \\
(\%, w / w)\end{array}$ \\
\hline Product A & 3.127 & 6.8 & 0.1349 & 0.0043 \\
\hline Product B & 3.235 & 2.6 & 0.0528 & 0.0016 \\
\hline Product C & 3.126 & 3.0 & 0.0603 & 0.0019 \\
\hline
\end{tabular}

HPLC, high-performance liquid chromatography.

duction rate increased by about 50-70 a.u./g sample/min in all parts (Fig. 3B). As shown in Fig. 4, aging of the three products (especially, "surface layer" part) with UV-A resulted in the highest singlet oxygen generation (124-308a.u./g sample/min), followed by aging with solar spectrum (33135 a.u./g sample/min) and in the dark (23-106 a.u./g sample/ min). Generally, product A (average $=142.2 \mathrm{a} . u$. $/ \mathrm{g}$ sample/ $\mathrm{min}$ ) showed the highest amount of singlet oxygen production, whereas products B (average $=66.2 \mathrm{a} . \mathrm{u} . / \mathrm{g}$ sample $/ \mathrm{min}$,
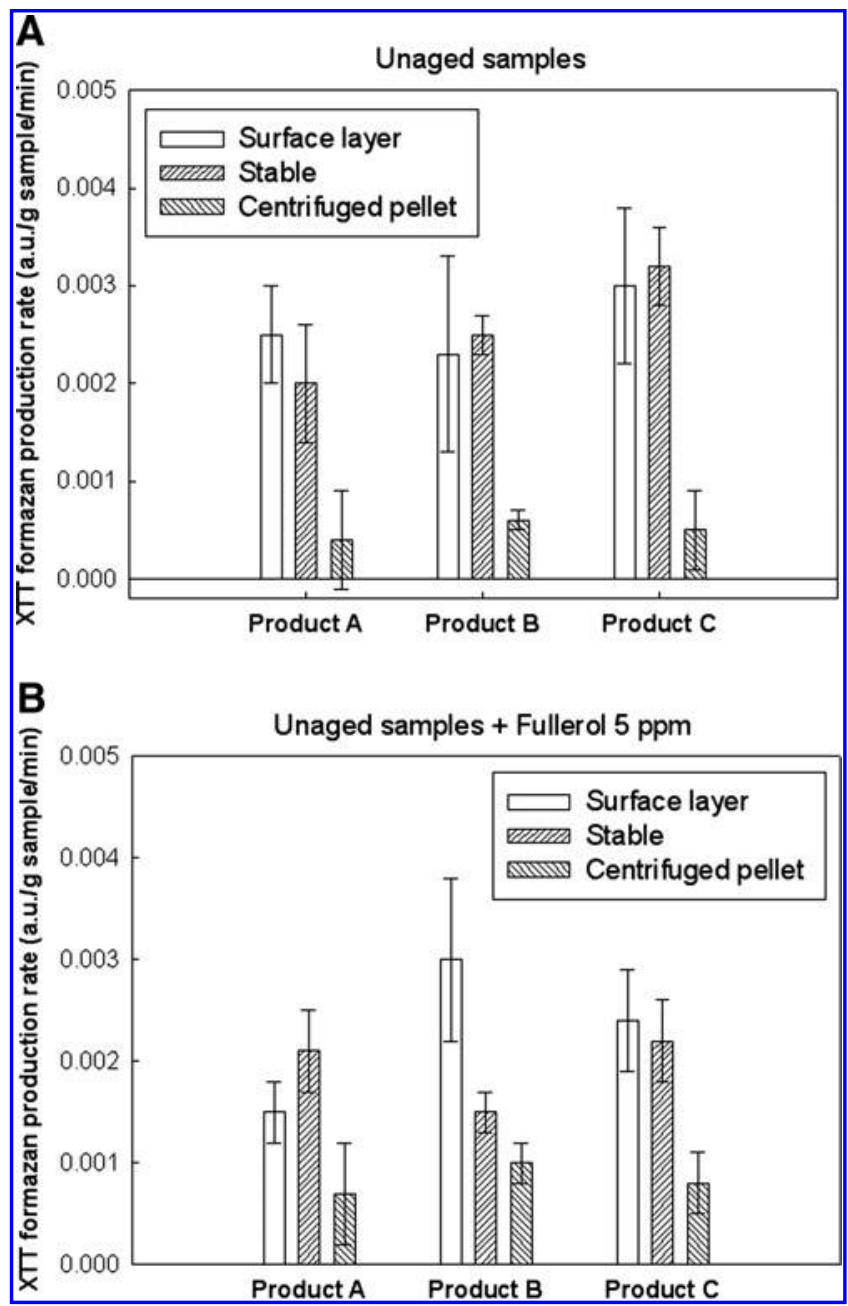

FIG. 1. Superoxide generation of unaged face creams (A, control) and unaged samples spiked with fullerol at $5 \mathrm{mg} / \mathrm{L}$ (B). XTT, 2,3-bis(2-methoxy-4-nitro-5-sulfophenyl)-2H-tetrazolium-5-carboxanilide. $p<0.005$ when compared with product A) and C (average $=53.8 \mathrm{a} . \mathrm{u} . \mathrm{g}$ sample $/ \mathrm{min}, p<0.005$ when compared with product A) appeared to generate smaller and comparable amounts of singlet oxygen. Figure 3B demonstrates that adding a known amount of fullerol, a known ${ }^{1} \mathrm{O}_{2}$ producer (Pickering

\section{A}

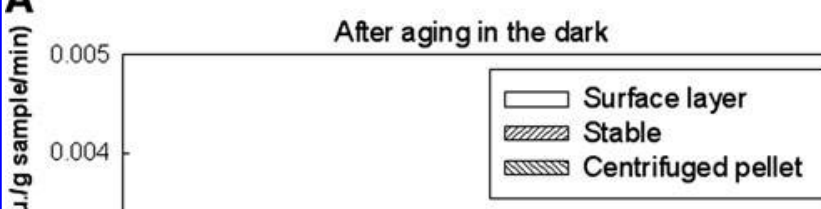

흔

ב⿱艹
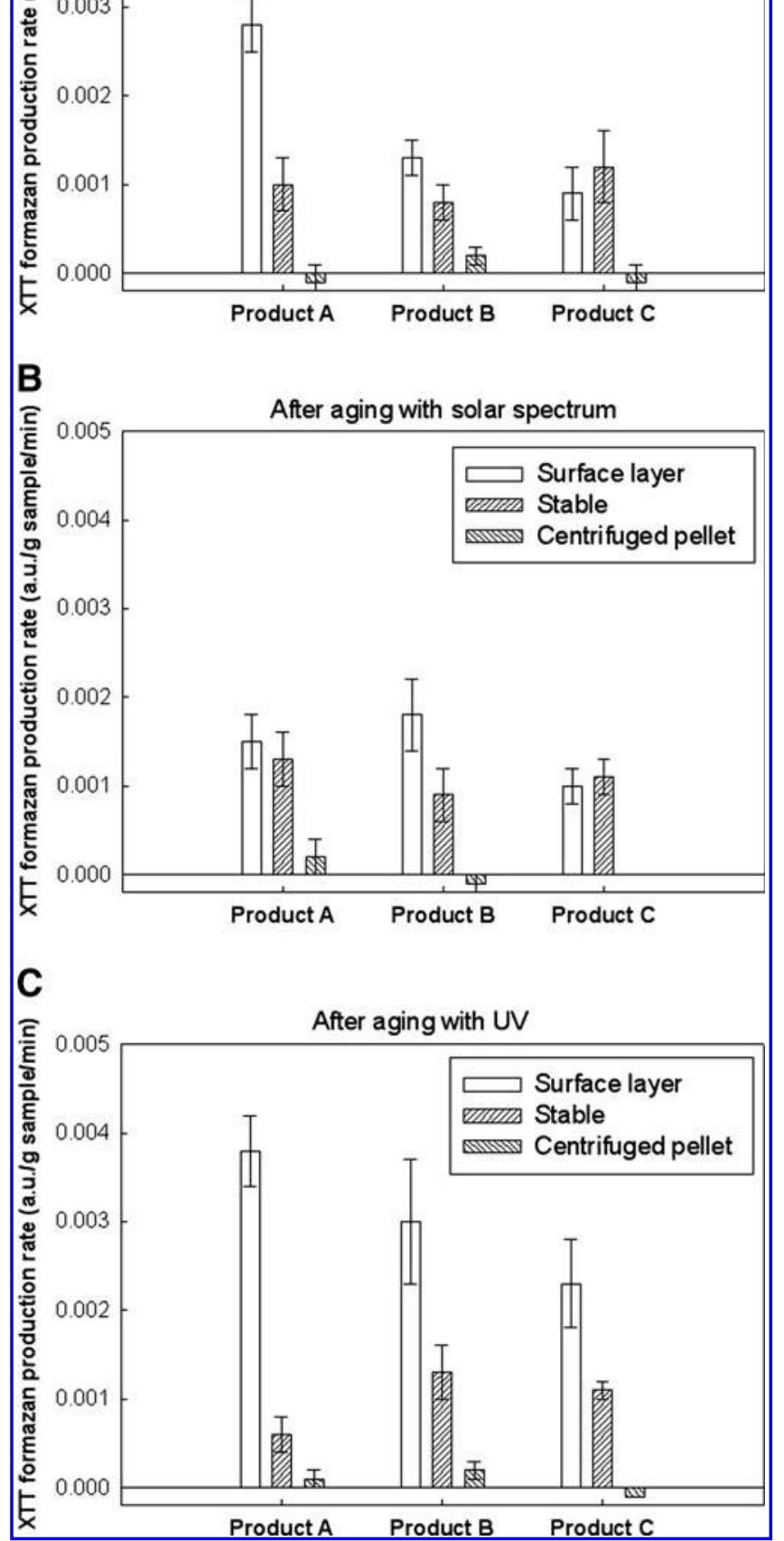

FIG. 2. Superoxide generation of aged face creams in the dark (A) and with solar spectrum (B) and UV (C). UV, ultraviolet. 


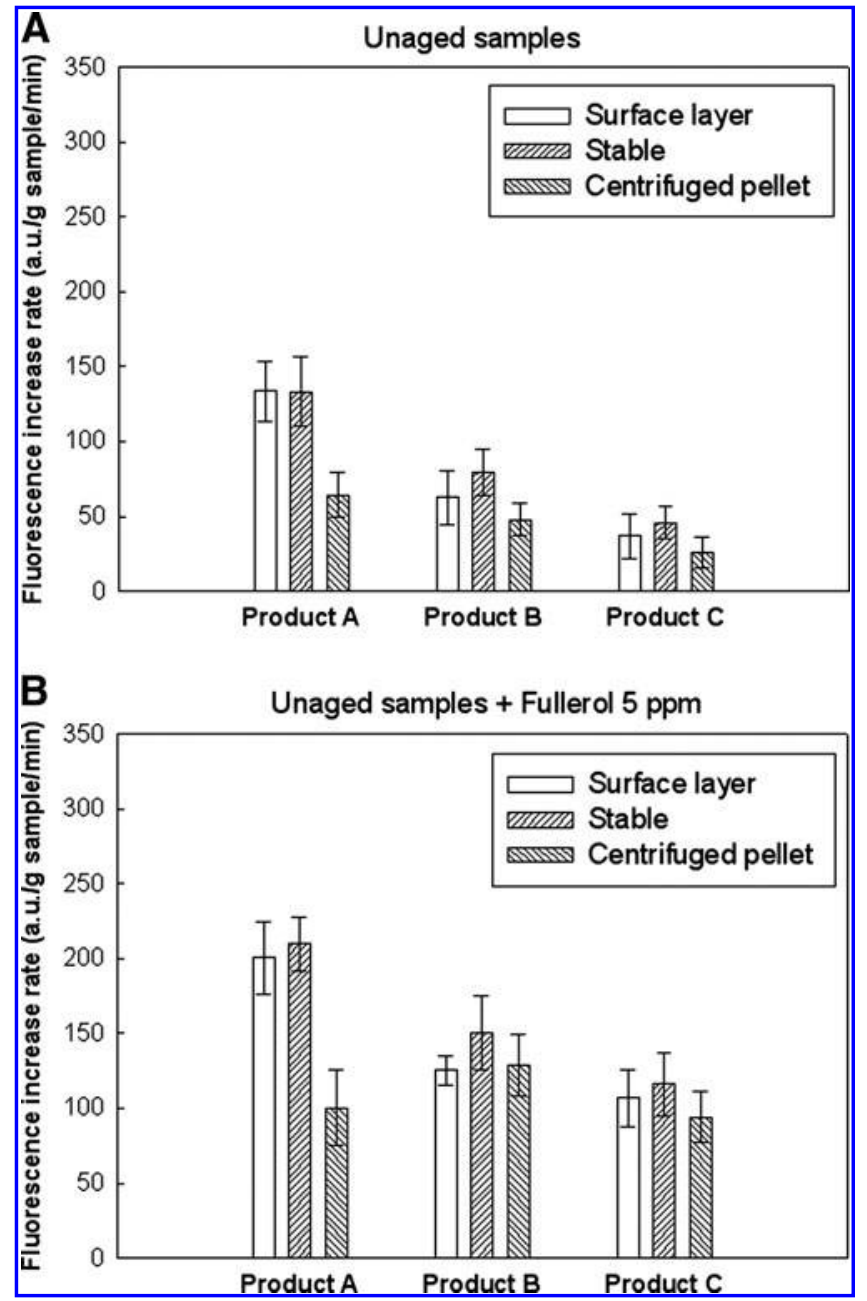

FIG. 3. Singlet oxygen production of unaged face creams (A, control) and unaged samples spiked with fullerol at $5 \mathrm{mg} / \mathrm{L}(\mathbf{B})$.

and Wiesner, 2005; Hotze et al., 2008; Chae et al., 2009a), to the unaged cream product results in lower generation rates than the aged product A in Fig. 4.

We postulate that the breakdown of other molecules contained within these cosmetics, either typical of cosmetics (e.g., thickeners, emulsifiers, and plasticizers) or more likely antioxidants (e.g., green tea extract, SOD, and others), during the aging process allows for increase of singlet oxygen production detected by the SOSG. This hypothesis would also explain why the unaged creams show less singlet oxygen production (Fig. 3A) than the aged samples (Fig. 4). Alternatively, the breakdown of these products could lead to photoactive molecules not present in the unaged samples (Roscher et al., 1994; Huong et al., 2008).

From Fig. 4, we determined that the highest generation rate of singlet oxygen (a.u./g sample/min) from product A (surface layer or stable part) is $0.03 \%$ of that generated by fullerol (Fig. 5). The cream type and aging conditions altered the amount of singlet oxygen produced by the creams and was significantly above levels produced by control samples. Moreover, the surface layer and stable suspension displayed similar levels of singlet oxygen generation, whereas the

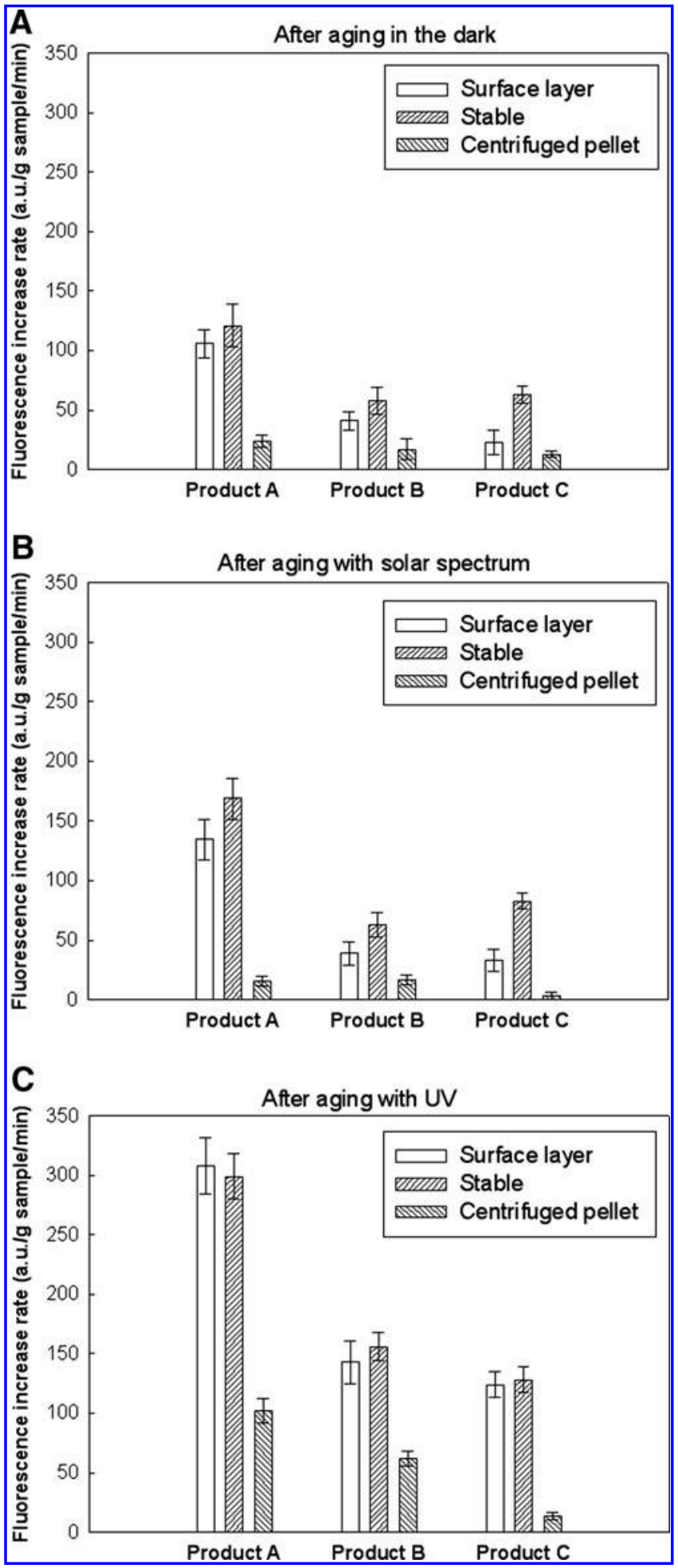

FIG. 4. Singlet oxygen production of aged face creams in the dark (A) and with solar spectrum (B) and UV (C).

centrifuged pellet part had the lowest production of singlet oxygen regardless of aging processes or product tested. The presence of amphiphilic molecules such as stearic acid may act as de facto surfactants, keeping fullerene molecules suspended in water. This result implies that the parts of the aged cream with the highest potential for ROS generation also have 


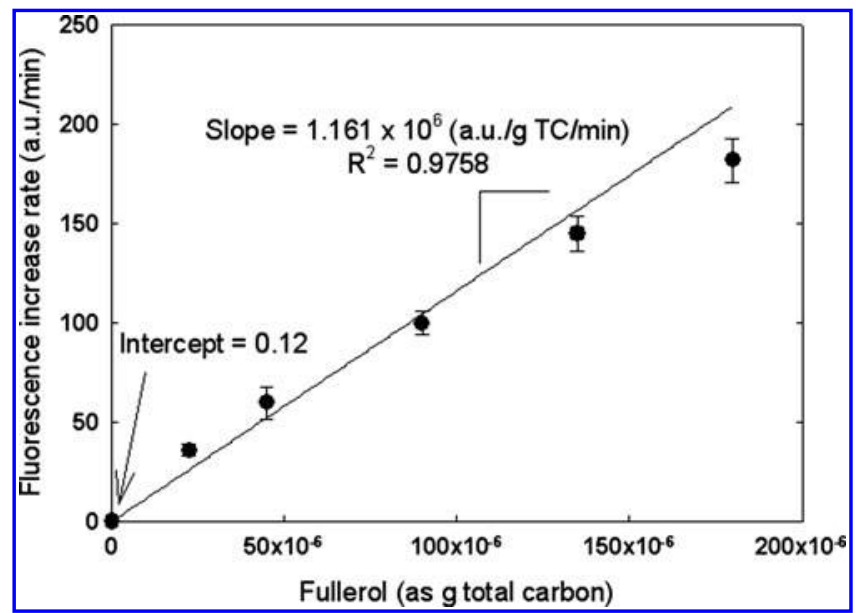

FIG. 5. Singlet oxygen production of fullerol in deionized water.

the highest colloidal stability. Another possible but admittedly tenuous observation is that product $\mathrm{A}$ contains approximately twice the amount of $\mathrm{C}_{60}$ than product $\mathrm{B}$ or $\mathrm{C}$ (Table 3) and produces approximately twice the singlet oxygen when aged under UV light (Fig. 4).

\section{Conclusions}

From SEM-EDX, FTIR, and ${ }^{13} \mathrm{C}$ NMR analyses, it was found that the quantity of fullerene in face creams was undetectably low for these analytical instruments and methodologies. In the case of SEM-EDX, fullerene peaks could not be separated from other organic ingredients in a carbon spectrum. For FTIR and ${ }^{13} \mathrm{C}$ NMR, fullerene peaks were not distinct because they were either below detection limits of the instruments or obscured by the presence of other molecules. Extraction by toluene followed by HPLC analysis, however, detected small quantities of fullerene at levels of $<0.005 \%$ $(\mathrm{w} / \mathrm{w})$. And we observed that ROS production of a cosmetic product (product A) containing fullerene is only $0.03 \%$ of that generated by fullerol nanoparticles.

Nanoengineered materials are likely to find numerous applications in commercial products because of their novel properties. Fullerene $\mathrm{C}_{60}$, added in creams as an antiaging and antioxidant agent, is one example. The unknown and untested possibility of direct effects of the fullerene materials on consumers remains an outstanding issue. But, unintended effects on the environment could result from the electron affinity and photochemistry of the molecule if it remains reactive over time in the aqueous environment. Detection of a significant amount of singlet oxygen production in the aged cream products raises several issues about the use of fullerene materials. Although radical scavenging may occur in the dark, it would appear that photochemically generated ROS would produce the opposite effect of that intended by adding fullerenes to these creams. We also conclude that the portions of the aged cream with the most transport potential (those remaining in the surface layer and stable suspension) are also the most photochemically active fractions. When cream byproducts are discharged into wastewater streams and ultimately water environments, higher mobility could lead to significant impacts of residual chemically active fullerenes. However, our ability to manipulate and fully exploit the direct relationship between fullerenes present in the products and ROS generation is limited. Further investigation of ROS generation by these and other aged products will improve our understanding of how their degradation will impact the environment.

\section{Acknowledgments}

This material is based upon work supported by the National Science Foundation (NSF) and the Environmental Protection Agency (EPA) under NSF Cooperative Agreement EF-0830093, Center for the Environmental Implications of NanoTechnology. The authors thank Dr. Hiroshi Yamamura at Hokkaido University and Armand Masion at CEREGE, Aix-Marseille University for help with SEM-EDX and ${ }^{13} \mathrm{C}$ NMR analysis, respectively. Additionally, the authors acknowledge support from Dr. Fabio Ziarelli and the Spectropole, the Analytical Facility of Aix-Marseille University, by allowing special access to the instruments purchased with European funding (FEDER OBJ2142-3341).

\section{Author Disclosure Statement}

No competing financial interests exist. Any opinions, findings, conclusions, or recommendations expressed in this article are those of the authors and do not necessarily reflect the views of the NSF or the EPA. This work has not been subjected to EPA review and no official endorsement should be inferred.

\section{References}

Badireddy, A.R., Hotze, E.M., Chellam, S., Alvarez, P., and Wiesner, M.R. (2007). Inactivation of bacteriophages via photosensitization of fullerol nanoparticles. Environ. Sci. Technol. $41,6627$.

Bartosz, G. (2006). Use of spectroscopic probes for detection of reactive oxygen species. Clin. Chim. Acta. 368, 53.

Bouchard, D., and Ma, X. (2008). Extraction and high-performance liquid chromatographic analysis of C-60, C-70, and [6,6]-phenyl C-61-butyric acid methyl ester in synthetic and natural waters. J. Chromatogr. A. 1203, 153.

Chae, S.R., Hotze, E.M., and Wiesner, M.R. (2009a). Evaluation of the oxidation of organic compounds by aqueous suspensions of photosensitized hydroxylated-C-60 fullerene aggregates. Environ. Sci. Technol. 43, 6208.

Chae, S.R., Wang, S.Y., Hendren, Z.D., Wiesner, M.R., Watanabe, Y., and Gunsch, C.K. (2009b). Effects of fullerene nanoparticles on Escherichia coli K12 respiratory activity in aqueous suspension and potential use for membrane biofouling control. I. Membr. Sci. 329, 68.

Chen, Z., Westerhoff, P., and Herckes, P. (2008). Quantification of C-60 fullerene concentrations in water. Environ. Toxicol. Chem. 27, 1852.

Cosmetic Dermatology Inc. (2009). Lineless Cream. Miami, FL. Available at: www.drbrandtskincare.com/p/lineless/linelesscream.

Fang, J.S., Lyon, D.Y., Wiesner, M.R., Dong, J.P., and Alvarez, P.J.J. (2007). Effect of a fullerene water suspension on bacterial phospholipids and membrane phase behavior. Environ. Sci. Technol. 41, 2636.

Flors, C., Fryer, M.J., Waring, J., Reeder, B., Bechtold, U., Mullineaux, P.M., Nonell, S., Wilson, M.T., and Baker, N.R. (2006). Imaging the production of singlet oxygen in vivo using a new fluorescent sensor, Singlet Oxygen Sensor Green. J. Exp. Botany 57, 1725. 
Fortner, J.D., Lyon, D.Y., Sayes, C.M., Boyd, A.M., Falkner, J.C., Hotze, E.M., Alemany, L.B., Tao, Y.J., Guo, W., Ausman, K.D., Colvin, V.L., and Hughes, J.B. (2005). $\mathrm{C}_{60}$ in water: nanocrystal formation and microbial response. Environ. Sci. Technol. 39, 4307.

Hotze, E.M., Labille, J., Alvarez, P., and Wiesner, M.R. (2008). Mechanisms of photochemistry and reactive oxygen production by fullerene suspensions in water. Environ. Sci. Technol. 42, 4175.

Huong, S.P., Rocher, E., Fourneron, J.D., Charles, L., Monnier, V., Bun, H., and Andrieu, V. (2008). Photoreactivity of the sunscreen butylmethoxydibenzoylmethane (DBM) under various experimental conditions. I. Photochem. Photobiol. A. Chem. 196, 106.

Isaacson, C.W., Kleber, M., and Field, J.A. (2009). Quantitative analysis of fullerene nanomaterials in environmental systems: a critical review. Environ. Sci. Technol. 43, 6463.

Jehlicka, J., Frank, O., Hamplova, V., Pokorna, Z., Juha, L., Bohacek, Z., and Weishauptova, Z. (2005). Low extraction recovery of fullerene from carbonaceous geological materials spiked with C-60. Carbon 43, 1909.

Lyon, D.Y., Adams, L.K., Falkner, J.C., and Alvarez, P.J.J. (2006). Antibacterial activity of fullerene water suspensions: effects of preparation method and particle size. Environ. Sci. Technol. 40, 4360.

Lyon, D.Y., Brunet, L., Hinkal, G.W., Wiesner, M.R., and Alvarez, P.J.J. (2008). Antibacterial activity of fullerene water suspensions $\left(\mathrm{nC}_{60}\right)$ is not due to ROS-mediated damage. Nano Lett. 8, 1539.

Moussa, F., Pressac, M., Genin, E., Roux, S., Trivin, F., Rassat, A., Ceolin, R., and Szwarc, H. (1997). Quantitative analysis of C60 fullerene in blood and tissues by high-performance liquid chromatography with photodiode-array and mass spectrometric detection. L. Chromatogr. B. -Anal. Technol. Biomed. Life Sci. 696, 153.

Larme Naturelle (2009). Larme Naturelle Moisture Cream. Tokyo, Japan. Available at: www.l-n.jp/html/newpage.html? code $=74$.
Pickering, K.D., and Wiesner, M.R. (2005). Fullerol-sensitized production of reactive oxygen species in aqueous solution. Environ. Sci. Technol. 39, 1359.

Roberts, J.E., Wielgus, A.R., Boyes, W.K., Andley, U., and Chignell, C.F. (2008). Phototoxicity and cytotoxicity of fullerol in human lens epithelial cells. Toxicol. Appl. Pharmacol. 228, 49.

Roscher, N.M., Lindemann, M.K.O., Kong, S.B., Cho, C.G., and Jiang, P. (1994). Photodecomposition of several compounds commonly used as sunscreen agents. I. Photochem. Photobiol. A. Chem. 80, 417.

Sachinvala, N., Parikh, D.V., Sawhney, P., Chang, S., Mirzawa, J., Jarrett, W., and Joiner, B. (2007). Silver(I) antimicrobial cotton nonwovens and printcloth. Polymers Adv. Technol. 18, 620.

Treubig, J.M., and Brown, P.R. (2002). Analysis of $\mathrm{C}_{60}$ and $\mathrm{C}_{70}$ fullerenes using high-performance liquid chromatography-Fourier transform infrared spectroscopy. J. Chromatogr. A. 960, 135.

Tweden, K.S., Cameron, J.D., Razzouk, A.J., Holmberg, W.R., and Kelly, S.J. (1997). Biocompatibility of silver-modified polyester for antimicrobial protection of prosthetic valves. L. Heart Valve Dis. 6, 553.

Ukeda, H.H., Maeda, S.S., Ishii, T.T., and Sawamura, M.M. (1997). Spectrophotometric assay for superoxide dismutase based on tetrazolium salt 3'-1-(phenylamino)-carbonyl-3, 4tetrazolium]-bis(4-methoxy-6-nitro)benzenesulfonic acid hydrate reduction by xanthine-xanthine oxidase. Anal. Biochem. 251, 206.

Wiesner, M.R., Lowry, G.V., Alvarez, P., Dionysiou, D., and Biswas, P. (2006). Assessing the risks of manufactured nanomaterials. Environ. Sci. Technol. 40, 4336.

Xia, T., Kovochich, M., Brant, J., Hotze, M., Sempf, J., Oberley, T., Sioutas, C., Yeh, J.I., Wiesner, M.R., and Nel, A.E. (2006). Comparison of the abilities of ambient and manufactured nanoparticles to induce cellular toxicity according to an oxidative stress paradigm. Nano Lett. 6, 1794.

Yannoni, C.S., Johnson, R.D., Meijer, G., and Bethune, D.S. (1991). ${ }^{13} \mathrm{C}$ NMR study of the $\mathrm{C}_{60}$ cluster in the solid state: molecular motion and carbon chemical. J. Phys. Chem 95, 9. 